\title{
Sind Ihre Steuern bereits optimiert?
}

\section{Fragen zur beruflichen Vorsorge und Säule 3a}

\author{
Schöpfen Sie die gebundene Vorsorge (Säule 3a) bereits voll aus? \\ Max. Beitragsmöglichkeiten 2008: \\ Sie sind in einer Pensionskasse (BVG) versichert: $\quad$ Fr. 6365.- \\ Sie sind nicht in einer Pensionskasse (BVG) versichert: $\quad 20 \%$ des AHV-Einkommens, max. Fr. 31824.-
}

O JA O NEIN

Haben Sie Ihr BVG-Einkaufspotential ausgeschöpft?

O JA O NEIN

BVG-Einkäufe können voll vom steuerbaren Einkommen abgezogen werden. So können je nach

Einkommenshöhe und Wohnort Steuereinsparungen bis zu 45 \% des BVG-Einkaufes realisiert werden.

\section{Ist Ihr Vermögen steuerlich optimal angelegt?}

OJA O NEIN

Kapitalgewinne (z.B. Kurssteigerungen) sind steuerfrei, während Kapitalerträge (z.B. Zinsen) als Einkommen versteuert werden müssen. Mit den flexiblen Vorsorgelösungen der FMH Insurance Services können Sie Ihr Gesamtvermögen so aufteilen, dass Sie Ihre Erträge möglichst steueroptimiert erwirtschaften.

\section{Haben Sie überprüft, ob Ihre BVG-Sparbeiträge erhöht werden können? \\ O JA O NEIN \\ Bei einer Erhöhung der BVG-Sparbeiträge werden grundsätzlich auch neue Einkaufspotentiale geschaffen, die Sie zur Steueroptimierung nutzen können. Bei den standeseigenen Versicherungslösungen können Sparbeiträge bis $25 \%$ des versicherten Lohnes vereinbart werden.}

\section{Verfügen Sie über mehrere Säule-3a-Anlagen?}

O JA O NEIN

Die Auszahlung einer Säule-3a-Anlage unterliegt einer einmaligen Kapitalleistungssteuer, bei der der Tarif mit zunehmender Höhe ansteigt. Verfügen Sie z. B. über zwei Säule-3a-Anlagen, die Sie in zwei verschiedenen Steuerperioden beziehen können, zahlen Sie durchschnittlich dank eines tieferen Satzes weniger Steuern.

\section{Verfügt Ihr Ehegatte/Ihre Ehegattin auch über ein BVG und eine Säule 3a?

Der in der Praxis mitarbeitende Ehepartner erhält für seine Arbeit oft keinen Lohn und ist daher auch im BVG nicht mitversichert. Durch das Bezahlen eines Lohnes kann der Ehepartner jedoch auch im BVG mitversichert werden und zudem für sich eine Säule 3a abschliessen.

\section{Auswertung}

$0 \times$ NEIN: Herzlichen Glückwunsch, Sie haben die wichtigsten Steueroptimierungen bereits vorgenommen. Gerne können wir aber überprüfen, ob Sie von den besten Lösungen profitieren.

1-6× NEIN: Sie verfügen über zusätzliches Optimierungspotential. Wir empfehlen Ihnen noch dieses Jahr eine Beratung durch einen FMH Insurance Services Spezialisten.

\section{Antworttalon}

Vorname / Name

Adresse

PLZ / Ort

Geburtsdatum

Telefon Privat/Geschäft

Beste Zeit für einen Anruf

E-Mail-Adresse

Ich wünsche eine persönliche Beratung.

\section{Bitte rufen Sie mich an.}

Ich interessiere mich für folgende Produkte:
O Säule $3 a$
O Pensionskasse BVC
O Finanz-/Steuerplanung
O Rechtsschutzversicherung
O Pensionsplanung
O Berufshaftpflichtversicherung

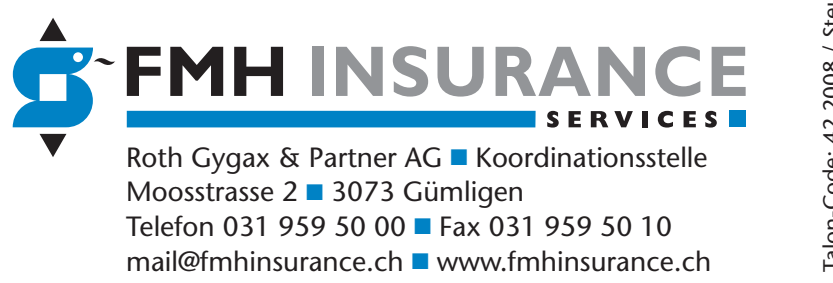

